were all significantly decreased in C group compared with the $S$ group; the levels in IB group were all significantly higher than those in $\mathrm{C}$ group. Atrial tissue from $\mathrm{C}$ group showed a large amount of interstitial fibrosis distributed throughout the tissue, evidenced by Masson staining, Sirius Red Staining and the expression of collagen-I by immunohistochemistry analysis. The expression of CT-1 in immunofluorescence analysis and Realtime PCR were all significantly higher in C group than those in $S$ group $(p<0.05)$, while decreased in IB group $(p<0.05)$, which was consistent with the changes of AT1R, and ACE.

Conclusions The expression of CT-1 significantly increased in atrial fibrosis in a canine model of atrial fibrillation, whereas irbesartan could decrease its expression. The results indicated that irbesartan could relieve atrial interstitial fibrosis, which was probably associated with the change of CT-1 expression.

\section{[gw22-e0770] ROLE OF CARDIOTROPHIN-1 IN A CANINE MODEL OF ATRIAL FIBRILLATION AND THE EFFECT OF IRBESARTAN ON CARDIOTROPHIN-1}

Han Hongya, Zhou Yujie, Bu Congya, Fang Zhe, Yan Zhenxian, Ma Hanying, Liu XiaoliBeijing Anzhen Hospital, Capital Medical University, Beijing, China

\subsection{6/heartjnl-2011-300867.71}

Objective Atrial fibrillation (AF) is one of the most common arrhythmias. The atrial interstitial fibrosis, is favourable for the occurrence and persistence of AF, which concerned with the Renin-Angiotensin System (RAS). We aim to study the dynamic changes of CT-1 both at mRNA and protein level in the atrium and the relationship between those changes and atrial interstitial fibrosis.

Methods Twenty two Male healthy canines were randomly divided into 3 groups: Sham group (S group, $n=6$ ), model group (control, C group, $\mathrm{n}=8$ ), and pacing+irbesartan treatment group (IB group, $n=8$ ). Masson staining and Sirius Red Staining were used to analyse collagen accumulation. Immunohistochemistry analysis of collagen-I, immunofluorescence analysis of CT-1 were done in the study. The mRNA level of CT-1 and angiotensin receptor type 1 (AT1R), angiotensin-converting enzyme (ACE) of atrium were all determined by Realtime-PCR analysis, respectively. Trans-thoracic echocardiography (TTE) was performed at baseline and after rapid pacing.

Results There are six canines in each group at the end of the study. After eight weeks of rapid atrial pacing, all the canines in C group, no canines in S group, and two canines in IB group were subjected to atrial fibrillation-both spontaneous and induced by burst stimulation. The diameter of the left atrium and the right atrium and left ventricular ejection fraction (LVEF) 